\title{
Factors predictive of prognosis after esophagectomy for squamous cell cancer
}

\author{
Houhuai Li, MD, PhD, ${ }^{\mathrm{a}}$ Qingzhen Zhang, ${ }^{\mathrm{a}}$ Lin Xu, MD, ${ }^{\mathrm{a}}$ Yijiang Chen, ${ }^{\mathrm{b}}$ Yongxiang Wei, ${ }^{\mathrm{c}}$ and Guoren Zhou, MS
}

Objective: To evaluate the prognosis after esophagectomy for squamous cell carcinoma of the thoracic esophagus and its prognostic factors.

\begin{abstract}
Methods: Six hundred five patients with primary squamous cell carcinoma of the thoracic esophagus who underwent curative esophagectomy between June 1997 and June 1998 were collected from 3 medical centers. Among them, 26 patients died from the operation and 26 patients did not complete adjuvant treatment owing to toxicity. Univariate and multivariate analysis was performed to identify prognostic factors for survival. The effect of adjuvant treatment on survival was also evaluated.

Results: The 1-, 3-, 5-, and 10-year overall survivals of 605 patients were $90 \%, 65 \%, 36 \%$, and $8 \%$, respectively. Multivariate analysis identified the following as independent prognostic factors: number of lymph node metastases $(P<.001)$, histologic differentiation $(P<.001)$, tumor location $(P=.002)$, depth of invasion $(P=$ $.020)$, and vascular invasion $(P=.023)$.

Conclusions: Several pathologic characteristics of the primary tumor are correlated with the outcome of esophagectomy for squamous carcinoma of the thoracic esophagus. Patients with fewer than 2 metastatic nodes after curative esophagectomy have a better prognosis than those with multiple involved nodes $(>2)$. To stratify patients appropriately for prognosis, it is necessary to refine the current 6th edition TNM staging system.
\end{abstract}

In China, there is an increased prevalence of esophageal cancer, most of which consists of squamous cell carcinoma (SCC). This is in contrast to the dominant trend toward adenocarcinoma in the Western world. The long-term outcomes for selected patients undergoing multimodality treatment, which combines surgery with chemotherapy and radiation, are promising. In this study, we collected data on 605 patients who had received multimodality therapy for esophageal cancer from 3 regional medical centers and retrospectively evaluated outcomes to identify prognostic factors leading to improved survival. ${ }^{1}$

\section{PATIENTS AND METHODS}

From June 1997 to June 1998, 712 patients with primary SCC of the thoracic esophagus were admitted to our regional thoracic surgical centers (Jiangsu Province Tumor Hospital, Jiangsu Province People's Hospital [Nanjing Medical University] and Nanjing Jiangbei People's Hospital [Dongnan University]). Among them, 605 patients underwent en bloc radical esophagectomy through a left thoracotomy, with a 2-field lymph node

\footnotetext{
From the Division of Thoracic Surgery, Department of Surgery, Jiangsu Province Tumor Hospital ${ }^{\mathrm{a}}$; the Division of Thoracic Surgery, Department of Surgery, Jiangsu Province People's Hospital ${ }^{\mathrm{b}}$; the Division of Thoracic Surgery, Department of Surgery, Nanjing Jiangbei People's Hospital ${ }^{\mathrm{c}}$; and the Division of Chemoradiotherapy, Department of Medicine, Jiangsu Province Tumor Hospital, ${ }^{\mathrm{d}}$ Nanjing, China.

Emphasis study of Jiangsu Province Cancer Hospital (ZK200703). Technology development project of Nanjing (YKK0463). Development Program of Technology Agency of Jiangsu Province (BS2006005).

Received for publication Jan 4, 2008; revisions received April 15, 2008; accepted for publication May 10, 2008.

Address for reprints: Houhuai Li, MD, PhD, Division of Thoracic Surgery, Department of Surgery, Jiangsu Province Tumor Hospital, Beiziting 42\#, Nanjing, China (E-mail: lihouhuai@vip.163.com).

J Thorac Cardiovasc Surg 2009;137:55-9

$0022-5223 / \$ 36.00$

Copyright (C) 2009 by The American Association for Thoracic Surgery doi:10.1016/j.jtcvs.2008.05.024
}

dissection in the mediastinum and upper abdomen. Reconstruction consisted of a gastric tube placed through the posterior mediastinum, and esophagogastrostomy was performed via a left cervical incision.

Postoperatively, the pathologic status of the resected tumors was evaluated to determine the need for adjuvant treatment. Patients with superficial cancer and no lymph node involvement underwent no further treatment. Those with tumor invading past the submucosa or with lymph node involvement were routinely administered chemotherapy and radiation. Chemotherapy consisted of 2 cycles: a bolus administration of cisplatin $\left(20 \mathrm{mg} / \mathrm{m}^{2}\right.$ per day) occurred on days 1 to 3 , and fluorouracil $\left(500 \mathrm{mg} / \mathrm{m}^{2}\right.$ per day) was given as a continuous infusion over 24 hours on days 1 to 5 . Postoperative radiotherapy was administered by linear accelerators with 10- to 15-MV photons. Radiotherapy was simulated to encompass a tumor volume with $5-\mathrm{cm}$ cephalocaudal margins and $2-\mathrm{cm}$ radial margins. Treatment ports were designed to encompass enlarged regional nodes and metastatic nodal beds based on preoperative computed tomographic evaluation and postoperative pathologic examination. Radiation was delivered in daily fractions of $1.8 \mathrm{~Gy}$ with a total dose of 50 to $60 \mathrm{~Gy}$ using a multiple-field technique.

Pathologic examination of the resected surgical specimens followed a standardized protocol. First, 4- $\mu \mathrm{m}$ sections of the tumor were stained with hematoxylin and eosin. The total number of lymph nodes resected and the number of lymph nodes involved with tumor were recorded. Vascular invasion was defined as infiltration of vessels or the presence of tumor emboli. Resection was considered microscopically incomplete when tumor cells were present less than $1 \mathrm{~mm}$ from the plane of resection (ie, the circumferential margin). The degree of tumor differentiation was classified as well, moderately, and poorly differentiated. Tumor staging occurred according to the pTNM system established by the International Union Against Cancer (UICC) in 2002 (6th edition). ${ }^{1}$

Postoperative patients were surveyed every 3 months by physical examination, every 6 months by imaging (computed tomographic scan and abdominal ultrasound), and every year by esophagoscopy. The study protocol was approved by the regional institutional review board. All patients provided written informed consent.

\section{Statistical Methods}

Continuous data were presented as mean values with standard deviations (mean $\pm \mathrm{SD}$ ). Means were compared with the Mann-Whitney test. 


\section{Abbreviations and Acronyms \\ $\mathrm{pM}=$ presence of metastasis \\ $\mathrm{pN} \quad=$ lymph node metastases \\ pT $=$ depth of tumor invasion \\ $\mathrm{SCC}=$ squamous cell carcinoma \\ $\mathrm{UICC}=$ International Union Against Cancer}

Categorical data were compared with a $\chi^{2}$ test (with the Yates correction) or the Fisher exact test. Follow-up continued until December 2007 or death if earlier. Overall survival was evaluated by the Kaplan-Meier method and included perioperative deaths. Overall survivals were compared by the $\log$-rank test. Multivariate analyses were performed by Cox regression. To evaluate the optimal cutoff point for the number of involved nodes as a predictor for survival, we delineated the relationship between the number of positive nodes and survival using a scatterplot of the variable versus Martingale residuals from a Cox proportional hazards regression model without the variable of interest. A smoothed fit of the scatter was then applied to detect the optimal cutoff point. ${ }^{2}$

All statistical tests were 2-sided. Statistical significance was set at the $5 \%$ level. Calculations were performed with SPSS version 12.0.1 (SPSS, Inc, Chicago, Ill).

\section{RESULTS}

A total of 605 patients (male/female: 512/93; age: $63.0 \pm$ 9.3 years) underwent en bloc radical esophagectomy. The median length of hospital stay was 15.8 days (range 10 156 days), and $26(4.3 \%)$ patients died as a result of the operation (male/female: $23 / 3$; age: $63.7 \pm 11.9$ years). Another 26 patients (male/female: $21 / 5$; age: $58.8 \pm 12.5$ years) did not complete adjuvant therapy owing to nephrotoxicity, gastrointestinal adverse reactions, and myelosuppression. Therefore, the other 553 patients (male/female: 468/85; age: $61.2 \pm 10.1$ years) completed the proposed chemoradiation treatment.

\section{Overall Survival}

Follow-up was complete for all 605 patients. The median follow-up was 47.0 months. At the time of this analysis, the $1-, 3-, 5-$, and 10-year overall survivals of the 605 patients were $90 \%, 65 \%, 36 \%$, and $8 \%$, respectively, with a median survival of 79.2 months.

The 1-, 3-, 5-, and 10-year disease-specific survivals for stage $0(\mathrm{n}=30)$ were $100 \%, 100 \%, 90 \%$, and $80 \%$; for stage $\mathrm{I}(\mathrm{n}=78), 99 \%, 95 \%, 85 \%$, and $58 \%$; for stage IIa $(\mathrm{n}=111), 99 \%, 88 \%, 65 \%$, and $34 \%$; for stage IIb $(\mathrm{n}=$ $165), 95 \%, 73 \%, 48 \%$, and $18 \%$; for stage III $(n=185)$, $92 \%, 67 \%, 44 \%$, and $9 \%$; and for stage IV $(\mathrm{n}=36)$, $85 \%, 35 \%, 19 \%$, and 0 (log-rank test; $P<0.001)$ (Figure 1).

\section{Factors Influencing Survival}

Apart from $\mathrm{pT}$ (depth of tumor invasion), $\mathrm{pN}$ (lymph node metastases), and $\mathrm{pM}$ (presence of metastasis), univariate

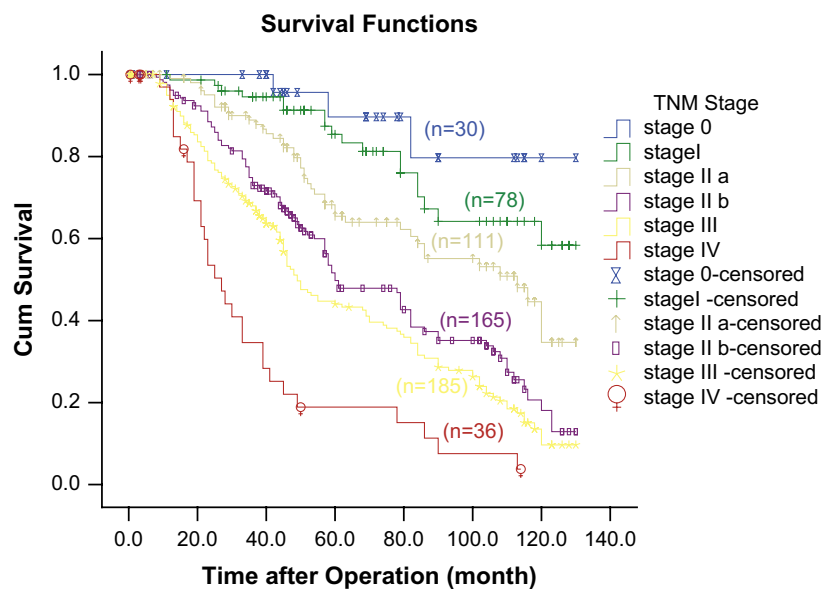

FIGURE 1. Cumulative disease-specific survival curves in terms of tumor pTNM stage. Median survival time for patients with stage $0(n=30)$, stage I $(\mathrm{n}=78)$, stage IIa $(\mathrm{n}=111)$, stage IIb $(\mathrm{n}=165)$, stage III $(\mathrm{n}=185)$, stage IV $(\mathrm{n}=36)$ were $130.0,130.0,112.4,60.8,49.7$, and 26.2 months, respectively (log-rank test, $P<.001)$.

analysis identified the following variables as significant predictors of survival: tumor location, histologic differentiation, vascular invasion, and number of lymph node metastases (Table 1). Age, gender, tumor length, total number of

TABLE 1. Clinicopathologic features influencing disease-specific survival of 605 patients

\begin{tabular}{|c|c|c|c|c|c|c|}
\hline \multirow[b]{2}{*}{ Variable } & \multirow[b]{2}{*}{ No. } & \multicolumn{4}{|c|}{ Survival $(\%)$} & \multirow[b]{2}{*}{$P$} \\
\hline & & $1 \mathbf{y}$ & $3 \mathbf{y}$ & $5 y$ & $10 y$ & \\
\hline \multicolumn{7}{|l|}{ Depth of invasion* } \\
\hline pTis & 30 & 100 & 100 & 90 & 80 & \multirow[t]{5}{*}{$<.001$} \\
\hline pT1 & 139 & 98 & 90 & 74 & 51 & \\
\hline pT2 & 171 & 96 & 77 & 57 & 29 & \\
\hline pT3 & 164 & 94 & 68 & 42 & 8 & \\
\hline pT4 & 101 & 88 & 58 & 37 & 8 & \\
\hline \multicolumn{7}{|l|}{ Tumor location } \\
\hline Upper & 175 & 92 & 59 & 33 & 13 & \multirow[t]{3}{*}{$<.001$} \\
\hline Middle & 227 & 96 & 78 & 57 & 23 & \\
\hline Lower & 203 & 96 & 86 & 68 & 31 & \\
\hline \multicolumn{7}{|c|}{ Histologic differentiation } \\
\hline Well & 164 & 96 & 88 & 75 & 44 & \multirow[t]{4}{*}{$<.001$} \\
\hline Moderate & 159 & 94 & 79 & 62 & 32 & \\
\hline Poor & 189 & 94 & 69 & 41 & 13 & \\
\hline Undifferentiated & 93 & 94 & 55 & 29 & 0 & \\
\hline \multicolumn{7}{|c|}{ Vaso-invasive growth } \\
\hline Not marked & 364 & 96 & 79 & 58 & 31 & \multirow[t]{2}{*}{$<.01$} \\
\hline Marked & 241 & 92 & 69 & 49 & 11 & \\
\hline \multicolumn{7}{|l|}{$\begin{array}{c}\text { No. of lymph node } \\
\text { metastases }\end{array}$} \\
\hline 0 & 156 & 99 & 93 & 77 & 43 & \multirow[t]{4}{*}{$<.001$} \\
\hline $1 \sim 2$ & 239 & 97 & 82 & 63 & 23 & \\
\hline 3 & 87 & 91 & 74 & 41 & 0 & \\
\hline$>3$ & 123 & 88 & 39 & 12 & 0 & \\
\hline
\end{tabular}

*Classification of primary tumor and TNM staging according to TMN classification (6th edition, 2002). 


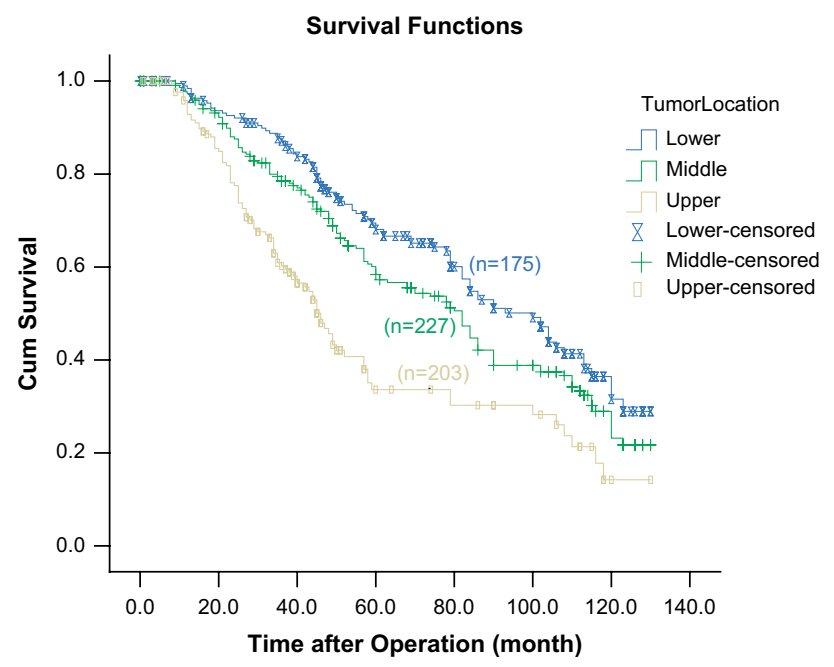

FIGURE 2. Cumulative disease-specific survival curves in terms of tumor location. Median survival time for patients with upper thoracic cancer ( $\mathrm{n}=$ $175)$, middle thoracic esophageal cancer $(n=227)$, and lower thoracic esophageal cancer $(\mathrm{n}=203)$ were $45.9,82.2$, and 93.8 months, respectively (log-rank test, $P<.001)$.

dissected lymph nodes, and time of operation did not influence the survival (data not shown).

Cumulative disease-specific survival curves in terms of tumor location, histologic differentiation, and vascular invasion are shown in Figures 2, 3 and 4, respectively. So that the optimal cutoff point for the number of positive lymph nodes could be determined, the Martingale residuals of the Cox model were first calculated and then plotted against the number of positive nodes (not shown). The cutoff value was 3 positive nodes. Subdivision of $\mathrm{pN}$ stage into 4 groups based

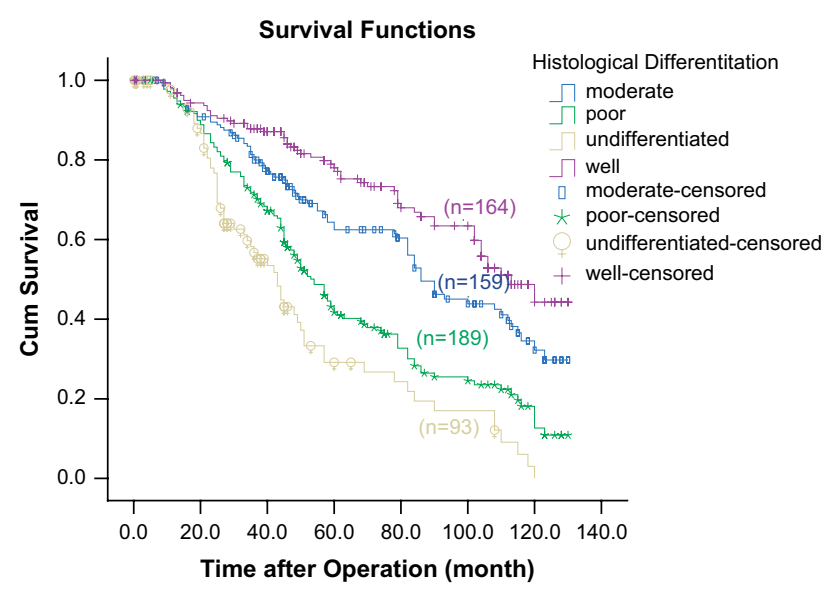

FIGURE 3. Cumulative disease-specific survival curves in terms of histologic differentiation of tumor. Median survival time for patients with well $(\mathrm{n}=164)$, moderate $(\mathrm{n}=159)$, poor $(\mathrm{n}=189)$, and undifferentiated ( $\mathrm{n}=93$ ) were $111.6,86.7,54.0$, and 43.1 months, respectively (log-rank test; $P<.001)$.

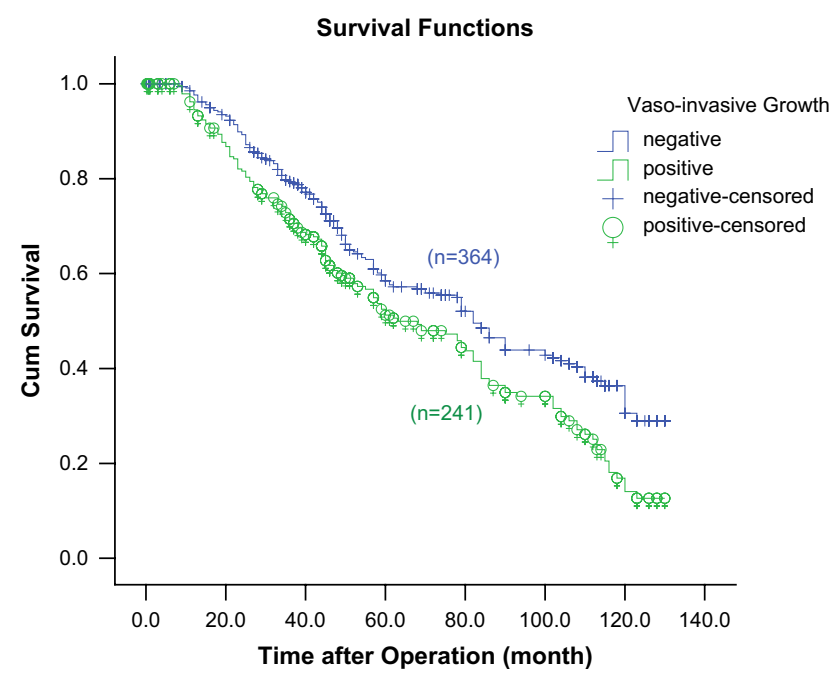

FIGURE 4. Cumulative disease-specific survival curves in terms of vasoinvasive growth of tumor. Median survival time for patients with negative $(\mathrm{n}=358)$ and positive $(\mathrm{n}=247)$ were 84.6 and 61.3 months, respectively (log-rank test; $P<.01)$.

on the number of positive nodes $(0,1-2,3$, and $\geq 4$ nodes positive) showed significant differences in disease-specific survival. For these groups, median survival times were $119.7(\mathrm{n}=156), 90.4(\mathrm{n}=239), 51.6(\mathrm{n}=87)$, and 32.7 ( $\mathrm{n}=123$ ) months, respectively (log-rank, $P<.001)$. Figure 5 shows that patients with 2 involved lymph nodes or fewer have a longer disease-specific survival than do those with more significant nodal disease ( $\geq 3$ positive nodes).

Multivariate analysis identified the following as independent prognostic factors: the number of lymph node metastases $(P<.001)$, histologic differentiation $(P<0.001)$, tumor location $(P=.002)$, depth of invasion $(P=.020)$, and vascular invasion $(P=.023)$ (Table 2$)$.

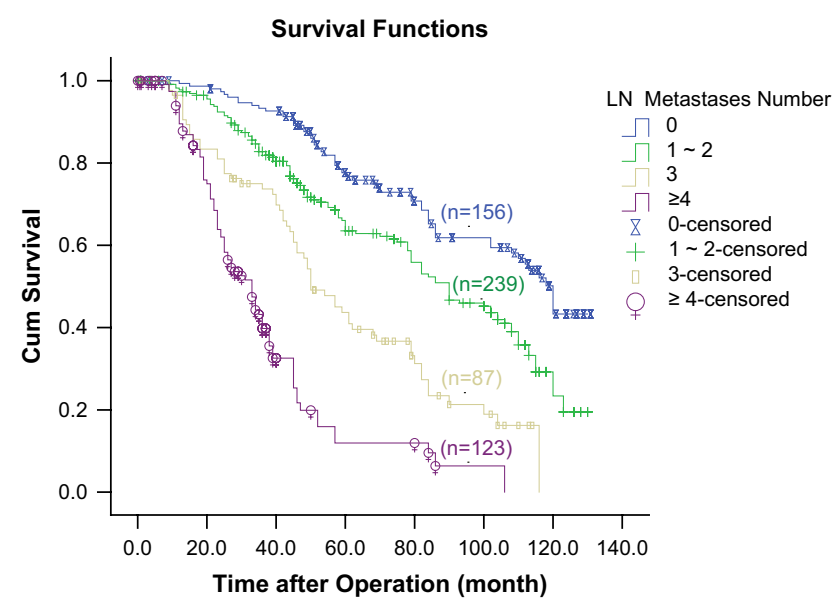

FIGURE 5. Cumulative disease-specific survival curves in terms of number of lymph node $(L N)$ metastases (log-rank test; $P<.001)$. 
TABLE 2. Multivariate analysis of prognostic factors for diseasespecific survival by the Cox proportional hazard model

\begin{tabular}{lcccc}
\hline \multicolumn{1}{c}{ Parameter* } & Coefficient & \multicolumn{1}{c}{$\boldsymbol{P}$} & Hazard & $\mathbf{9 5 \% ~ C I}$ \\
\hline Tumor location & -.243 & .002 & .784 & $0.674-0.911$ \\
Depth invasion & .183 & .020 & 1.201 & $1.030-1.401$ \\
Involved lymph node & .373 & $<.001$ & 1.453 & $1.359-1.553$ \\
Histologic differentiation & .502 & $<.001$ & 1.653 & $1.480-1.845$ \\
Vasoactive growth & .261 & .023 & 1.298 & $1.037-1.625$ \\
\hline CI, Confidence interval. *Parameters are categorized in Table 1. &
\end{tabular}

Additional multivariate analysis was performed, including the TNM stage of the disease as a covariate. We excluded the pT factor and the number of involved lymph nodes for a more appropriate analysis of independent prognostic factors. In this additional analysis, vascular invasion no longer appeared as an independent prognostic factor for increased survival $(P=.073)$.

To investigate the role of postoperative chemoradiation on survival after esophagectomy, we excluded the 26 patients who died perioperatively. The remaining 579 patients were divided into 3 groups: those who completed adjuvant treatment after surgery $(n=449)$, those who did not complete adjuvant treatment $(n=26)$, and those for whom adjuvant treatment was not recommended owing to early disease $(n=104)$. Figure 6 shows the improved survival in the group who completed adjuvant treatment, in contrast to that in the group who did not complete treatment.

\section{DISCUSSION}

Radical esophagectomy has become the standard surgical procedure to achieve an accurate pathologic staging for pa-

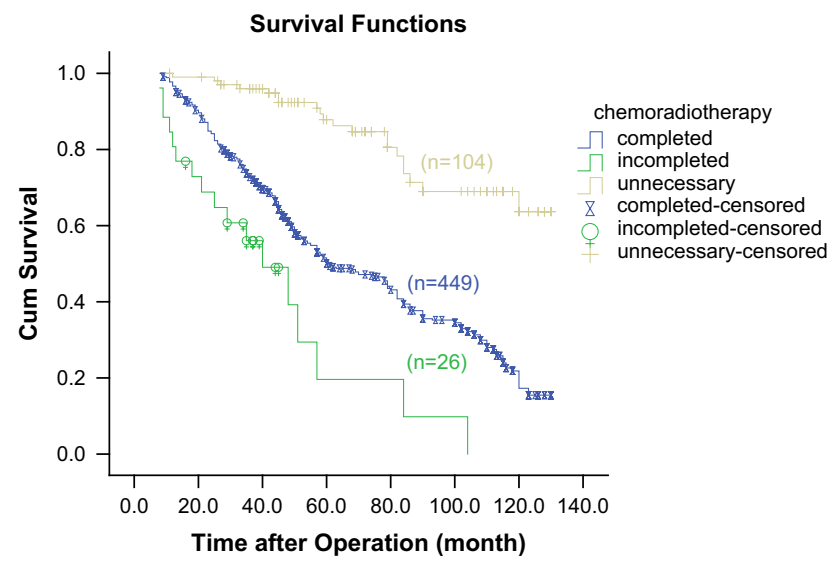

FIGURE 6. Cumulative disease-specific survival curves in terms of postoperative adjuvant radiochemotherapies for the patients with SCC of the thoracic esophagus $(\mathrm{n}=579)$. The 1-, 3-, 5- and 10-year disease-specific survivals for the unnecessary postoperative radiochemotherapy patients (n $=104$ ) were $99 \%, 96 \%, 88 \%$, and $63 \%$; for the completed postoperative radiochemotherapy patients $(\mathrm{n}=449)$ were $95 \%, 72 \%, 49 \%$, and $17 \%$; and for the incompleted patients $(\mathrm{n}=26)$ were $77 \%, 56 \%, 20 \%$, and 0 ; respectively (log-rank [Mantel-Cox]; $P<.001)$. tients with esophageal cancer, ${ }^{3,4}$ but the long-term outcomes are not necessarily satisfactory according to the UICC-TNM staging. ${ }^{4,5}$ In this study, we collected a well-defined homogeneous cohort of 605 patients with SCC of the thoracic esophagus and evaluated the long-term outcomes to identify prognostic factors. Given that the data were retrospective, our conclusions are limited. Our findings are interpreted within the context of existing published data.

\section{Esophageal Cancer Located in the Upper Thorax Is an Unfavorable Prognostic Factor}

One hundred twenty-nine of 175 patients with upper thoracic cancer who died had locoregional recurrences in the upper mediastinum despite extensive lymphadenectomy. Locoregional recurrence consisted of progression of mediastinal nodal disease, including direct invasion into the tracheobronchial trees. More meticulous lymphadenectomy around the tracheobronchial trees and bilateral recurrent laryngeal nerves may be important to improve the outcomes of these patients. ${ }^{3}$

\section{Patients with Esophageal Cancer Achieved Improved Survival after Multimodality Therapy}

All 605 patients underwent left thoracotomy with 2-field lymph node dissection in the mediastinum and upper abdomen. Including those patients who underwent adjuvant chemoradiation, the 3- and 5-year overall survivals were $65 \%$ and $36 \%$. Previous studies in the existing literature show excellent results with a surgical approach that includes 3-field lymphadenectomy. Such an approach likely has the highest chance of tumor clearance, ${ }^{5,6}$ but this is at the expense of increased morbidity and mortality and poorer postoperative quality of life. ${ }^{4}$ Our study suggests that patients who successfully completed adjuvant treatment had an increased survival compared with those who did not complete the treatment. Randomized studies with larger cohorts of patients are needed to further evaluate the effect of adjuvant chemoradiation.

\section{UICC-TNM Staging of Esophageal Cancer Needs to be Refined}

The TNM system for classifying the anatomic extent of disease in cancer has been in existence for more than 50 years. In this study, we evaluated additional prognostic factors in a homogeneous group of patients with esophageal cancer treated by multimodality therapy. Our study confirmed that the $\mathrm{pT}$ and $\mathrm{pN}$ stages according to the 6th edition UICC-TNM staging model are indeed important predictors of survival.

However, there is ongoing debate about the need for revision of the current guidelines, driven by surgeons who believe that the current system does not accurately stratify patients for prognosis. ${ }^{7-9}$ Recently, the UICC-TNM committee decided to establish guidelines for the submission and evaluation of proposed changes to the TNM system. ${ }^{10}$ 
In our study, other pathologic characteristics of the primary tumor, including tumor location, histologic differentiation, and vascular invasion, were shown to correlate with outcome. Such pathologic criteria as histologic differentiation and vascular invasion represent the biological property of the tumor and should be considered in the modified staging system.

Moreover, survival is influenced not only by the presence or absence of involved lymph nodes (pN0 vs pN1) but also by the number of positive nodes. In our study, subdivision of pN1 tumors into $\mathrm{pN} 1 \sim 2, \mathrm{pN} 3$, and $\mathrm{pN} \geq 4$ had significant differences in survival. These findings are similar to observations made by Rice and associates, ${ }^{7}$ who subclassified their node-positive patients as N1 ( $\leq 2$ metastatic nodes) and $\mathrm{N} 2(\geq 3$ metastatic nodes) on the basis of significantly different survivals. Generally, patients with more advanced nodal disease had worse survival than those with less nodal disease (eg, $1-3$ vs $\geq 4,1-4$ vs $\geq 5$ ). ${ }^{5,11}$ Published studies thus far differ in the calculations by which the optimal cutoff for the number of positive nodes is determined. By calculating the Martingale residuals, we found that the cutoff should be 3 positive nodes. The patients with fewer than 2 metastatic nodes had improved long-term survival; this was an independent and favorable prognostic factor. Similar data have been reported for adenocarcinoma of the esophagus. ${ }^{12}$ Thus, patients with fewer than 2 metastatic nodes after esophagectomy have an improved prognosis compared with those with more advanced nodal disease. This suggests that more aggressive adjuvant therapy may be the key to improve the survival of patients with multiple lymph node metastases.

Within the group of patients with positive locoregional nodes, a further subdivision can be made on the basis of the number of positive nodes divided by the total number of resected nodes, also known as the lymph node ratio. ${ }^{13,14}$ Various lymph node ratios have been proposed ${ }^{13-15}$ owing to differences in the techniques of lymphadenectomy and the total number of resected nodes. ${ }^{2}$ In our patients, lymph node dissection includes the superior, middle, and inferior mediastinum and around the splenic, celiac, and hepatic arteries. About 10 to 17 nodes (a median number of 14) were resected for each patient. The total number of resected nodes was not associated with differences in overall survival. Thus this may suggest that lymph node ratio may not be an independent factor. However, more studies are needed to confirm this finding.

So that patients can be better stratified according to prognosis, the current 6th edition TNM staging system for esophageal cancer must be revised. We would suggest that pathologic criteria such as histologic differentiation and vascular invasion be incorporated into the new staging system inasmuch as our study showed these to be independent prognostic factors in patients who underwent multimodal treatment of esophageal cancer. We look forward to the modification of the UICC-TNM staging system with clinicopathologic variables that are confirmed to be independent prognosticators in further studies and large randomized trials.

\section{CONCLUSION}

Several pathologic characteristics of the primary tumor are correlated with differences in long-term outcomes after esophagectomy for SCC of the thoracic esophagus. Patients with fewer than 2 metastatic nodes after radical esophagectomy have a better prognosis than those with multiple involved nodes $(>2)$.

\section{References}

1. Sobin LH. TNM, sixth edition: new developments in general concepts and rules. Semin Surg Oncol. 2003;21:19-22.

2. Frank I, Blute ML, Leibovich BC, Cheville JC, Lohse CM, Kwon ED, et al. pT2 classification for renal cell carcinoma: can its accuracy be improved? J Urol. 2005; 173:380-4

3. Altorki NK, Skinner DB. Occult cervical nodal metastasis in esophageal cancer: preliminary results of three-field lymphadenectomy. J Thorac Cardiovasc Surg. 1997;113:540-4

4. Fujita H, Kakegawa T, Yamana H, Shima I, Toh Y, Tomita Y, et al. Mortality and morbidity rates, postoperative course, quality of life, and prognosis after extended radical lymphadenectomy for esophageal cancer. Comparison of three-field lymphadenectomy with two-field lymphadenectomy. Ann Surg. 1995;222:654-62.

5. Nishimaki T, Suzuki T, Suzuki S, Kuwabara S, Hatakeyama K. Outcomes of extended radical esophagectomy for thoracic esophageal cancer. J Am Coll Surg. 1998;186:306-12.

6. Matsubara T, Ueda M, Nagao N, Takahashi T, Nakajima T, Nishi M. Cervicothoracic approach for total mesoesophageal dissection in cancer of the thoracic esophagus. J Am Coll Surg. 1998;187:238-45.

7. Rice TW, Blackstone EH, Rybicki LA, Adelstein DJ, Murthy SC, DeCamp MM, et al. Refining esophageal cancer staging. J Thorac Cardiovasc Surg. 2003;125: 1103-13.

8. Korst RJ, Rusch VW, Venkatraman E, Bains MS, Burt ME, Downey RJ, et al. Proposed revision of the staging classification for esophageal cancer. $J$ Thorac Cardiovasc Surg. 1998;115:660-9; discussion 669-70.

9. Rusch VW. Should the esophageal cancer staging system be revised? J Thorac Cardiovasc Surg. 2003;125:992-3.

10. Gospodarowicz MK, Miller D, Groome PA, Greene FL, Logan PA, Sobin LH, et al. The process for continuous improvement of the TNM classification. Cancer. 2004; $100: 1-5$.

11. Korst RJ, Ginsberg RJ, Ailawadi M, Bains MS, Downey RJ Jr, Rusch VW, et al Proposed revision of the staging classification for esophageal cancer. $J$ Thorac Cardiovasc Surg. 1998;115:660-70.

12. Hölscher AH, Bollschweiler E, Bumm R, Bartels H, Höfler H, Siewert JR, et al. Prognostic factors of resected adenocarcinoma of the esophagus. Surgery. 1995; 118:845-55

13. Saha S, Dehn TC. Ratio of invaded to removed lymph nodes as a prognostic factor in adenocarcinoma of the distal esophagus and esophagogastric junction. Dis Esophagus. 2001;14:32-6.

14. Eloubeidi MA, Desmond R, Arguedas MR, Reed CE, Wilcox CM. Prognostic factors for the survival of patients with esophageal carcinoma in the U.S.: the importance of tumor length and lymph node status. Cancer. 2002;95:1434-43.

15. Hagen JA, DeMeester SR, Peters JH, Chandrasoma P, DeMeester TR. Curative resection for esophageal adenocarcinoma: analysis of 100 en bloc esophagectomies. Ann Surg. 2001;234:520-30; discussion 530-1. 